\title{
An RCS Measurement Technique to Extract the Impedance of High-Impedance Aperture and Slot Antennas
}

\author{
Reza Azadegan*, Kamal Sarabandi \\ The University of Michigan, Radiation Lab \\ Ann Arbor, MI 48109-2122 USA \\ FAX: $+1-734-747-2106$ \\ email: azadegan@eecs.umich.edu
}

\section{ABSTRACT}

In this paper, a novel technique to measure the input impedance of generally high-impedance aperture antennas are introduced whereby a relationship is established between the RCS of a slot/apreture antenna to its Impedance/conductance. Then, the necessary condition which must be met when the receiving antenna is terminated by a matched load is derived and used to identify the corresponding RCS. Comparing the RCS of the loaded antenna in conjunction with the criteria provided by the proposed method can determined whether the receiving antenna is matched or not. If not, one can change the termination resistance till the required conditions on the RCS are met, and then, identify the matched load; that is equal to the impedance of the antenna at resonance.

\section{INTRODUCTION}

In the wake of recent interests in MEMS and nanotechnology, there is an ongoing effort towards the application of the above-mentioned technologies in fabrication of different devices, components and circuits. One severe concern is the high impedance of these devices. The high-impedance property originates from the small size of these devices. Also, it is crucial to maintain the current level extremely low so as to obtain micro-power systems. On the other hand, testing high impedance devices at RF is not possible using currently available microwave measurement systems designed for $50 \Omega$. The problem stems from the fact that the impedance of the microwave transmission lines can not go very much higher than $50 \Omega$, and even so, they become very lossy and start to radiate.

MEMS resonators/filters, for example, are very high-impedance $(\approx 10 K \Omega$ ), and thus, extremely hard to measure. Moreover, they require high impedance antennas and RF circuits to be integrated with. Another example, indicating the importance of high-impedance antennas and their measurement issues, is the idea of high impedance miniature antennas matched to high-impedance electronics and or nano-devices.

In what follows, a resonant half-wave slot antenna is considered. Although the center fed slot antenna is not so high impedance as of MEMS or nano-devices, its impedance is still an order of magnitude larger then standard transmission line. Furthermore, its radiated and scattered fields can be analytically calculated using duality and Babinet principle. 

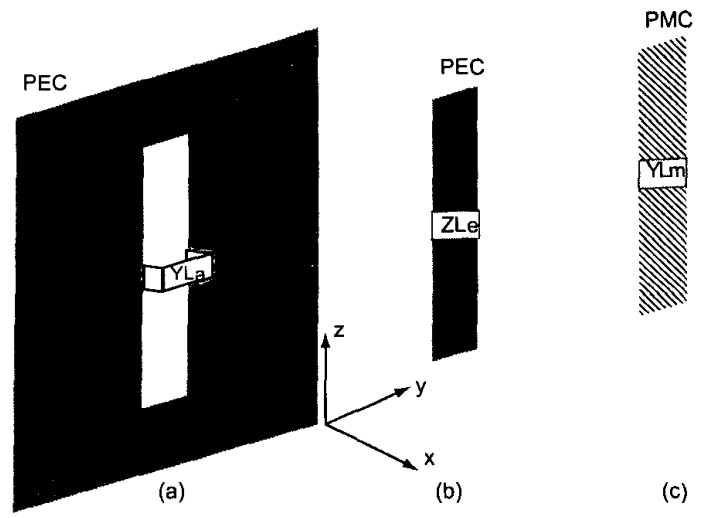

Figure 1: Illustration of Babinet's principle for three receiving antennas (a): an aperture antenna in a PEC screen, (b): the complimentary PEC strip, (c): the dual case of PMC strip.

\section{FORMULATION}

Consider an infinite perfectly conducting screen with a narrow half-wavelength aperture as shown in Fig. 1(a), and its complimentary structure consisting of a thin strip of PEC in Fig. 1(b).A thin strip of perfectly magnetic conducting strip is shown in Fig. 1(c). The objective of the proposed measurement technique is to infer the impedance of the aperture antenna from its measured RCS. First, we need to calculate the RCS of the aperture antenna of Fig. 1 (a) as a function of the load admittance of the aperture $\left(Y_{L a}\right)$. Note that the scattering from the aperture antenna has two components; 1) the reflection from an often large PEC plate, and 2 ) the scattering from the aperture itself. The scattering from the plate is much larger than the perturbation introduced by the slot. Therefore, it may supersede the perturbation introduced by the aperture, and also reduce the sensitivity of the measurements. The effect of the ground plane, however, can be calculated analytically, and then subtracted from the total scattered fields. This corresponds to the coherent subtraction in the radar measurement experiment. Assuming that the structure of Fig. 1(a) is illuminated by a normal incident plane wave of the form of $\bar{H}^{i}=\hat{z} H_{0} e^{j k x}$, the backscattered filed at $\theta=90^{\circ}$ can be written as [1]

$$
\bar{E}_{\text {total }}^{s}=\bar{E}_{\text {plate }}^{s}+\bar{E}_{\text {slot }}^{s} \text { where } \bar{E}_{\text {plate }}^{s}=-j \eta k L_{x} L_{y} H_{0} \frac{e^{-j k r}}{2 \pi r} \hat{a}_{\theta} \quad \Rightarrow \sigma_{\text {plate }}=\frac{1}{\pi}\left|k L_{x} L_{y}\right|^{2}
$$

The contribution of the aperture/slot to the scattered field $\left(\bar{E}_{\text {siot }}^{s}\right)$ can be calculated using Babinet's principle. This scattered field is dual of the scattered field of the PEC strip of Fig. 1(b) when illuminated by a dual excitation, namely $-\frac{1}{n}{\overline{E_{0}}}^{i}$. In the same fashion, the scattered fields of PEC and PMC are dual, given dual excitations. Therefore, the scattered fields of a dipole can be calculated instead. Reciprocity implies the the scattered fields of a 


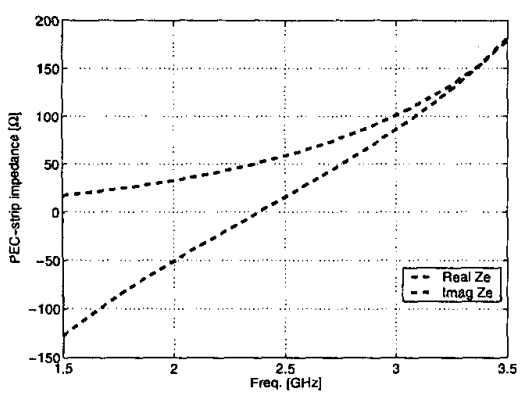

(a)

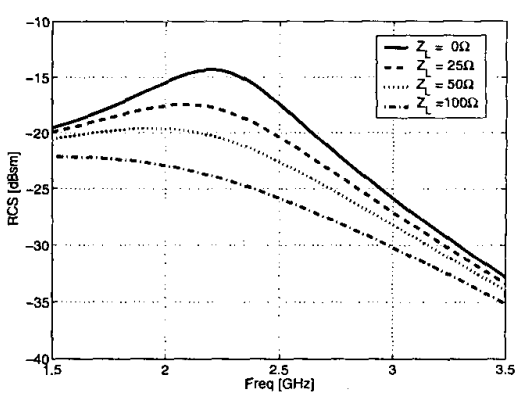

(b)

Figure 2: (a): The input impedance of the PEC strip $\left(Z_{e}\right)$; and (b): the maximum backscattering cross section of the printed dipole as a function of load resistance $\left(Z_{L}\right)$.

dipole has similar from to its radiated fields given by [2-4].

$$
\bar{E}_{e}^{s}=j \eta \frac{2 E_{0} / k}{Z_{e}+Z_{L e}} \frac{e^{-j k r}}{2 \pi r} \quad \Rightarrow \quad \sigma_{e}=\frac{4 \eta^{2}}{\pi k^{2}\left|Z e+Z_{L e}\right|^{2}}
$$

Equation (2) suggests that the RCS be maximum when $Z_{L e}=0$. When the load resistance is equal to the real part of the antenna impedance, the maximum RCS reduces by a factor of four (-6dB) as compared to its peak. The input impedance of the strip antenna of Fig. 1(b) with $\ell=5.5 \mathrm{~cm}$, as well as the variation of its maximum back-scattering cross-section as a function frequency when the load resistance has been varied as a parameter are shown in Fig. 2. Applying duality by changing $\bar{E}_{e}^{s} \rightarrow \eta \bar{H}_{a}^{s}$, and $Z_{e} \rightarrow \eta^{2} Y_{a}$, the scattered field due to the presence of the aperture found to be:

$$
\bar{E}_{a}^{s}=j \frac{2 E_{0} / k}{\eta\left(Y_{a}+Y_{L a}\right)} \frac{e^{-j k r}}{2 \pi r} \quad \Rightarrow \quad \sigma_{a}=\frac{4}{\pi k^{2} \eta^{2}\left|Y a+Y_{L a}\right|^{2}}
$$

then the total scattered field is

$$
\bar{E}_{\text {total }}^{s}=\bar{E}_{\text {plate }}^{s}+\bar{E}_{\text {slot }}^{s}=-j E_{0} \frac{e^{-j k r}}{2 \pi r}\left(k L_{x} L_{y}-\frac{2 E_{0} / k}{\eta\left(Y_{a}+Y_{L a}\right)}\right) \hat{a}_{\theta}
$$

which gives:

$$
\sigma_{t}=\frac{1}{\pi}\left|k L_{x} L_{y}-\frac{2}{k \eta\left(Y_{a}+Y_{L a}\right)}\right|^{2}
$$

\section{RESULTS AND CONCLUSIONS}

The total back-scattering $\sigma_{t}$ as in (5) is a quantity that can be directly measured and/or simulated. This quantity, however, contains a large clutter component, namely, the reflection from the plat, which does not carry any information about the aperture impedance, and so should be removed. One way to do so is to use coherent subtraction. The other simpler way is to subtract the scattered amplitude/power of the plate from that of the total backscattered 


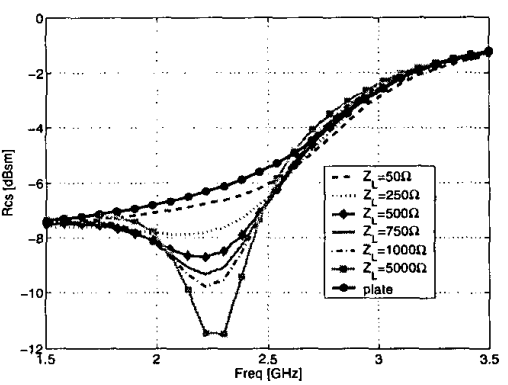

(a)

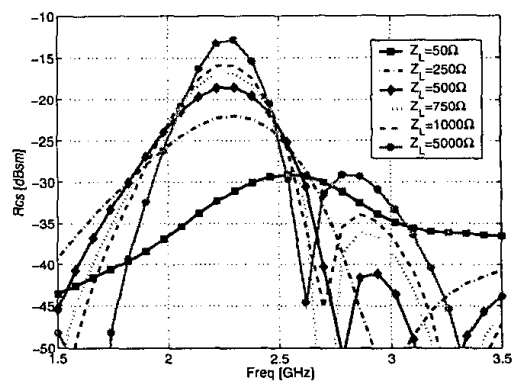

(b)

Figure 3: (a): Simulated back-scattering RCS of $\ell=5.5 \mathrm{~cm}$ slot antenna in a $14 \mathrm{~cm} \times 14 \mathrm{~cm}$ PEC screen [5]; (b). extracted RCS of the aperture for different values of load resistance $\left(Z_{L a}\right)$.

$\sigma_{t}$. Knowing that the normal incident plane wave excites both the plate and slot in-phase, one can conclude that the scattered fields are in-phase in so far as the admittance of the aperture is real. Thus, subtracting the amplitudes provides accurate results at resonance. Figure 3(a) shows the simulated RCS of a slot antenna with $\ell=5.5 \mathrm{~cm}$ with a ground plane of $14 \mathrm{~cm} \times 14 \mathrm{~cm}$ for different values of load impedance. As seen in this figure, the aperture perturbs the RCS. In Fig. 3(b) the effect of the plate is excluded, and the contribution of the aperture to the total scattered field is illustrated. For aperture antennas, the open load $Y_{L a}=0$, gives the maximum RCS, while a trace whose peak is $6 \mathrm{~dB}$ below the maximum peak corresponds to the matched load. Therefore, one can discern that the impedance of this antenna is about $500 \Omega$.

Using the same methods, The impedance of miniature slot antennas with much higher impedances $(\approx 10 K \Omega)$ can be accurately measured. Fabrication and testing of high impedance antennas, probes, and field sensors is an important step towards further advances towards emerging fields such as micro and nanotechnology.

\section{REFERENCES}

[1] C. A. Balanis Advanced Engineering Electromagnetics, John-Wiley, 1989.

[2] C. A. Balanis Antennas Theory Analysis and Design, 2nd edition John-Wiley, 1997.

[3] Y. HU, "Back-scattering cross section of a center-loaded cylinderical antenna," IRE Trans. Antennas and Propagat. vol. 6 pp. 140-148, Jan. 1958.

[4] K. M. Chen, V. Liepa. "The minimization of the Back scattering of a cylinder by central loading," IEEE Trans. Antennas and Propagat. vol. 12 pp. 576-582, Sep. 1964.

[5] IE3D, Electromagnetic design and simulation package, version 10.0 Zeland Software Corp. 